\title{
Prevalência e fatores de risco de hanseníase na ESF Palmeira do Açaí
}

\section{Hansen's disease: prevalence and risk factors in "ESF Palmeira do Açaí"}

DOI: $10.46919 / \operatorname{archv1n5-021}$

Recebimento dos originais: 10/07/2020

Aceitação para publicação: 30/08/2020

\section{Luna Varela do Carmo}

Médica especialista em Clínica Médica pelo Conjunto Hospitalar do Mandaqui (CHM). Instituição: Conjunto Hospitalar do Mandaqui (CHM). Endereço profissional: Rua Voluntários da Pátria, 4301.

CEP: 02401-400.

E-mail: luna.varela@hotmail.com

\section{Natália Rodrigues Eugênio}

Médica especialista em Clínica Médica pela Fundação Santa Casa de Misericórdia do Pará (FSCMPA). Instituição: Fundação Santa Casa de Misericórdia do Pará (FSCMPA).

Endereço: Rua Bernal do Couto, 988.

CEP: 66055-080.

E-mail: natalia-eugenio@hotmail.com

\section{Patrick Abdala F. Gomes}

Doutor em Biologia dos Agentes Infecciosos e Parasitários pela Universidade Federal do Pará (UFPA). Instituição: Centro Universitário do Pará (CESUPA).

Endereço: Av. Almirante Barroso, $\mathrm{n}^{\circ} 3775$.

CEP: 66613-903.

E-mail: patrick.gomes@prof.cesupa.br

\section{RESUMO}

Introdução: A hanseníase, causada pelo bacilo Mycobacterium leprae, sempre gerou um estigma social intenso. A transmissão ocorre por meio, principalmente, das vias aéreas superiores. Mesmo que os índices de prevalência da Hanseníase tenham apresentado uma vertiginosa e importante queda nos últimos 25 anos, a prevalência da doença ainda é alarmante.

Objetivo: A presente pesquisa tem como objetivo verificar a prevalência e fatores de risco de Hanseníase na área 0020, da ESF Palmeira do Açaí localizada no bairro de Águas Lindas, no município de Ananindeua, no Estado do Pará, no ano de 2012, entre os meses de Janeiro a Setembro.

Metodologia: Para a coleta de dados, foi realizada uma visita na Secretaria Municipal de Saúde de Ananindeua, para a obtenção dos relatórios do SIAB, e à UBS Nova Águas Lindas. Posteriormente, foi feita uma análise acerca dos dados retirados dos relatórios A1 e da ficha A, obtidos na visita às instituições citadas. Os aspectos observados na ficha A foram gênero, quantidade de cômodos, tratamento e abastecimento de água, tipo de casa e destino de dejetos.

Resultados: Foi analisada uma amostra de 1578 pessoas residentes na área 0020 da ESF Palmeira do Açaí. Foram detectados 4 casos de pacientes hansenianos. Desses, o gênero masculino predominou (75\%); 100\% bebem água não tratada, não possuem abastecimento de água e o destino de dejetos é a fossa; o tipo de casa predominante é de tijolo (75\%), com uma média de 4 cômodos/residência e 3 moradores/residência. Além disso, há laço consanguíneo entre 2 dos casos (50\%). Na área pesquisada, essa prevalência foi de $0,25 \%$. 
Conclusão: A prevalência de 0,25\% é considerada alta quando comparada ao Estado do Pará, onde é de 0,09\%. Recebe a classificação "hiperendêmica", corroborando os riscos a que são submetidos os moradores hansenianos da área, como casas com excesso de pessoas por cômodo. Confirmou-se a relevância da consanguinidade na transmissão, tendo sido observada em $50 \%$ dos casos.

Palavras-chave: prevalência, Hanseníase, Saúde Pública.

\begin{abstract}
Introduction: Hansen's disease, caused by the bacillus Mycobacterium leprae, has always caused an intense social stigma. Transmission occurs mainly through the upper airways. Even though the prevalence rates have shown a vertiginous and important drop in the last 25 years, the number of people affected is still alarming.

Objective: This research aims to verify the prevalence and risk factors of Hansen's disease in area 0020, of the ESF Palmeira do Açaí located in the neighborhood of Águas Lindas, in the municipality of Ananindeua, in the State of Pará, among the from January to September of 2012.

Methodology: For data collection, a visit was made to the Municipal Health Department of Ananindeua, to obtain reports from SIAB (primary care information system), and to UBS Nova Águas Lindas. Subsequently, an analysis was made about the data taken from Reports A1 and Form A, obtained during the visit to the mentioned institutions. The aspects observed in Form A were gender, number of rooms, water treatment and supply, type of house and destination of waste. Results: A sample of 1578 people living in area 0020 of the ESF Palmeira do Açaí was analyzed. Four cases of Hansen's disease patients were detected. Of these, the male gender predominated (75\%); 100\% drink untreated water, have no water supply and the destination of waste is the cesspool; the predominant type of house is brick (75\%), with an average of 4 rooms / residence and 3 residents / residence. In addition, there is a blood link between 2 of the cases (50\%). In the surveyed area, this prevalence was $0.25 \%$.

Conclusion: The prevalence of $0.25 \%$ is considered high when compared to the State of Pará, where it is $0.09 \%$. It receives the classification "hyperendemic", corroborating the risks to which the Hansen's inhabitants of the area are submitted, such as houses with an excess of people per room. The relevance of inbreeding in transmission was confirmed, having been observed in $50 \%$ of cases.
\end{abstract}

Keywords: prevalence, Hansen's disease, public health.

\title{
1 INTRODUÇÃO
}

Em 2008, o Brasil ocupou o segundo lugar mundial em número de casos de Hanseníase1. Segundo dados preliminares em janeiro de 2013, o coeficiente de prevalência da hanseníase no Brasil está em 1,49 casos por 10.000 habitantes e o número absoluto é de 28.919 casos no país 2 .

O Pará é o estado com maior contribuição de novos casos na região Norte do Brasil, seguido pelos estados de Rondônia, Tocantins, Amazonas, em ordem decrescente ${ }^{3}$.

Quanto ao número de internações por complicações e/ou sequelas da hanseníase, no ano de 2012, o estado do Pará foi responsável por 93 de 936 da região Norte e 5241 no país no mesmo período ${ }^{4}$.

A doença é causada pelo Mycobacterium leprae, um parasita intracelular obrigatório, de crescimento lento, não cultivado in vitro e que apresenta o espectro de formas clínicas que vão do polo 
tuberculóide (TT) ao Virchoviano (LL), que resultam da relação que se estabelece entre a resposta imune celular do hospedeiro e o parasita ${ }^{5}$. A doença sempre gerou um estigma social intenso, de acordo com a sua história evolutiva, e isso persiste, mesmo que em menor grau ${ }^{6}$.

As principais fontes de bactérias são provavelmente as mucosas das vias aéreas superiores. O bacilo de Hansen tem a capacidade de infectar grande número de indivíduos, no entanto, poucos adoecem. Essa propriedade não é função apenas das características intrínsecas da bactéria, mas depende, sobretudo, de sua relação com o hospedeiro e do grau de endemicidade do meio ${ }^{7}$.

Além disso, alguns estudos passaram a demonstrar associação significativa entre a ocorrência da doença e a presença atual e antiga de hanseníase entre parentes co-sangüíneos ${ }^{8}$.

Mesmo que os índices de prevalência da Hanseníase e suas complicações tenham apresentado uma vertiginosa e importante queda nos últimos 25 anos, devido à implantação de programas de políticas públicas e a introdução da poliquimioterapia, a prevalência da doença ainda é alarmante, segundo bases de dados do Ministério da Saúde.

\section{OBJETIVO}

A presente pesquisa tem como objetivo verificar a prevalência e fatores de risco de Hanseníase na área 0020, da ESF Palmeira do Açaí localizada no bairro de Águas Lindas, no município de Ananindeua, no Estado do Pará, no ano de 2012, entre os meses de Janeiro a Setembro.

\section{METOLODOGIA}

Para a coleta de dados, foi realizada uma visita na Secretaria Municipal de Saúde de Ananindeua, obtendo-se os relatórios do SIAB/SUS, assim como outra visita à Unidade Básica de Saúde Nova Águas Lindas.

Posteriormente, foi feita uma análise acerca dos dados retirados dos relatórios A1 (Consolidado do cadastramento familiar por microárea), da ficha A (Ficha para cadastramento das famílias) e ficha BHAN (Ficha para acompanhamento de pessoas com hanseníase), obtidos nas visitas às instituições citadas. Os aspectos observados na ficha A foram gênero, quantidade de cômodos, tratamento e abastecimento de água, tipo de casa e destino de dejetos.

\section{RESULTADOS}

Foi analisada uma amostra de 1578 pessoas residentes na área 0020 da ESF Palmeira do Açaí. Foram detectados 4 casos de pacientes hansenianos no período selecionado. Desses, o gênero masculino predominou (75\%); 100\% bebem água não tratada, não possuem abastecimento de água e o destino de 
dejetos é a fossa; o tipo de casa predominante é de tijolo (75\%), com uma média de 4 cômodos/residência e 3 moradores/residência. Além disso, há laço consanguíneo entre 2 dos casos (50\%). Na área pesquisada, essa prevalência foi de $0,25 \%$.

\section{CONCLUSÃO}

A prevalência de 0,25\% é considerada alta quando comparada ao Estado do Pará, onde é de 0,09\% ${ }^{9}$. Recebe a classificação "hiperendêmica", corroborando os riscos a que são submetidos os moradores hansenianos da área, como casas com excesso de pessoas por cômodo. Confirmou-se a relevância da consanguinidade na transmissão ${ }^{10,11}$, tendo sido observada em $50 \%$ dos casos.

\section{REFERÊNCIAS}

1 DIAS, Regina Célia and PEDRAZZANI, Elisete Silva. Políticas públicas na Hanseníase: contribuição na redução da exclusão social. Rev. bras. enferm. [online]. 2008, vol.61, n.spe, pp.753756. ISSN 1984-0446. https://doi.org/10.1590/S0034-71672008000700016.

2 Relatório Anual de Avaliação do PPA 2012-2015: ano base 2013. Ministério do Planejamento, Orçamento e Gestão. Secretaria de Planejamento e Investimentos Estratégicos. - Brasília: SPI/MP.

3 Silva FL, da Silva APL. Tendências da hanseníase no Tocantins, um estado hiperendêmico do Norte do Brasil, 2001-2012. Cad. Saúde Pública vol.31 no.5 Rio de Janeiro May 2015.

4 Portal da Saúde. Ministério da Saúde / DATASUS - Departamento de Informática do SUS. Consulta: SIH (morbidade por estado/município).

5 COHEN JM. Hanseníase ocular: uma abordagem histórica. Arq. Bras. Oftalmol. vol.72 no.5 São Paulo Sept./Oct. 2009.

6 Baialardi KS. O estigma da hanseníase: relato de experiência em grupo com pessoas portadoras. Hansen Int. 2007;32(1): 27-36.

7 Fundação Nacional de Saúde (BR). Guia de vigilância epidemiológica. 5a ed. Brasília: FUNASA; 2002.

8 SANTOS, Andréia Soprani dos; CASTRO, Denise Silveira de and FALQUETO, Aloísio. Fatores de risco para transmissão da Hanseníase. Rev. bras. enferm. [online]. 2008, vol.61, n.spe, pp.738-743. ISSN 1984-0446. https://doi.org/10.1590/S0034-71672008000700014.

9 De Freitas LRS, Duarte EC, Garcia LP. Análise da situação epidemiológica da hanseníase em uma área endêmica no Brasil: distribuição espacial dos períodos 2001 - 2003 e 2010 - 2012. Rev. bras. epidemiol. 20 (04) Oct-Dec. 
10 Moet FJ, Pahan D, Schuring RP, Oskam L, Richardus JH. Physical distance, genetic relationship, age, and leprosy classification are independent risk factors for leprosy in contacts of patients with leprosy. J Infect Dis 2006 Feb 1;193(3):346-53.

11 Durães SMB, Guedes LS, Cunha MD, Cavaliere FAM, Oliveira MLWDR. Estudo de 20 focos familiares de hanseníase no município de Duque de Caxias, Rio de Janeiro. An Bras Dermatol 80[Supl 3], S295-300. 2005. Ref Type: Journal (Full).

\section{ANEXO}

- MODELO DA FICHA “A” (SIAB/SUS)

Figura 1: Modelo da Ficha A (retirado do Manual do Sistema de Informação da Atenção Básica, 2000).
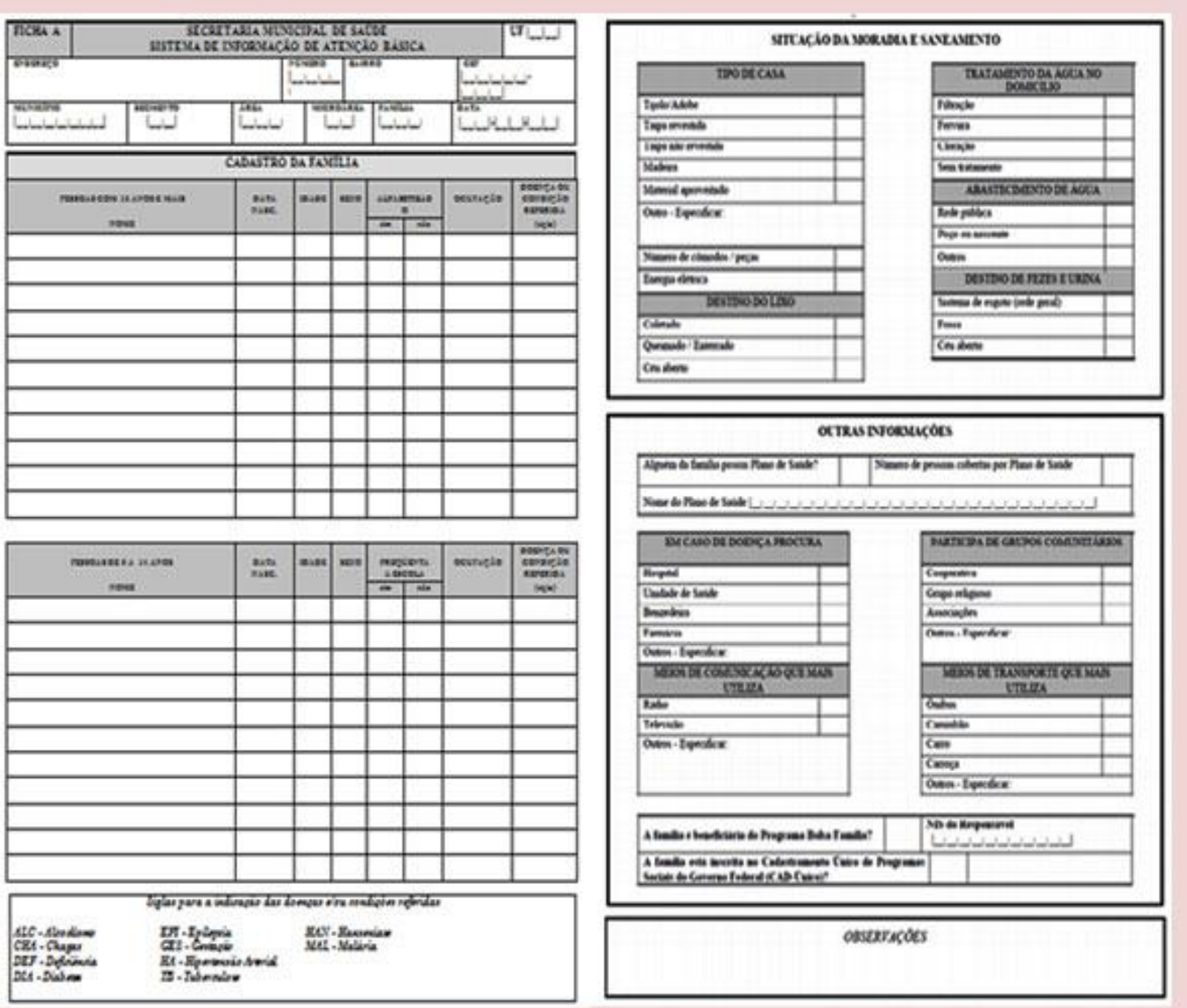